\title{
Efficacy of second-line treatment and importance of comorbidity scores and clinical parameters affecting prognosis in elderly patients with non-small cell lung cancer without epidermal growth factor receptor mutations
}

\author{
SUNG YONG LEE ${ }^{1 *}$, EUN JOO KANG ${ }^{2 *}$, SUK YOUNG LEE ${ }^{2 *}$, HONG JUN KIM ${ }^{2 *}$, \\ KYUNG HOON MIN $^{1 *}$, GYU YOUNG HUR ${ }^{1 *}$, JAE JEONG SHIM ${ }^{1 *}$, KYUNG HO KANG $^{1 *}$, \\ SANG CHEUL OH ${ }^{2 *}$, JAE HONG SEO ${ }^{2 *}$ and JUN SUK KIM ${ }^{2 *}$ \\ ${ }^{1}$ Division of Pulmonology, Department of Internal Medicine; ${ }^{2}$ Division of Medical Oncology, \\ Department of Internal Medicine, Korea University Guro Hospital, Seoul 08308, Republic of Korea
}

Received October 17, 2016; Accepted September 28, 2017

DOI: $10.3892 / \mathrm{ol} .2017 .7350$

\begin{abstract}
The present study investigated the importance of comorbidity scores and clinical parameters in elderly patients with non-small cell lung cancer (NSCLC) not harboring epidermal growth factor receptor (EGFR) mutations who received second-line chemotherapy. The present study also compared the efficacy of tyrosine kinase inhibitor and cytotoxic chemotherapy as second-line treatment in elderly patients. The present study retrospectively reviewed the treatment of elderly patients with NSCLC ( $\geq 70$ years old) who received second-line chemotherapy at Korea University Guro Hospital. Patients who had an EGFR mutation were excluded from the analysis. Between 2005 and 2013, 126 patients were included in the present study. The median progression-free survival (PFS) and overall survival (OS) for all patients who received second-line treatment were 2.47 months [ $95 \%$ confidence interval (CI), 2.08-2.86] and 8.63 months $(95 \% \mathrm{CI}$, 5.99-11.28), respectively. A total of 52 patients (41.3\%) were treated with tyrosine kinase inhibitor (TKI) and 74 (58.7\%) were treated with chemotherapy. No difference was observed in the median PFS and OS between the TKI and chemotherapy groups ( $\mathrm{P}=0.287$ for $\mathrm{PFS}$ and $\mathrm{P}=0.374$ for $\mathrm{OS})$. The Charlson comorbidity index was not associated with survival, whereas a simplified comorbidity score and clinical factors, including
\end{abstract}

Correspondence to: Dr Eun Joo Kang, Division of Medical Oncology, Department of Internal Medicine, Korea University Guro Hospital, 148 Gurodong-ro, Guro, Seoul 08308, Republic of Korea E-mail: kkangju11@naver.com

\section{*Contributed equally}

Key words: non-small cell lung carcinoma, epidermal growth factor receptor, second-line treatment, elderly, comorbidity, prognostic factors poor performance status, short PFS of first-line chemotherapy, presence of brain metastasis and low serum albumin and sodium levels were significant prognostic factors in these elderly patients. Second-line chemotherapy was not beneficial to patients who had at least 3 of these factors and a median OS of 1.73 months, whereas patients who had less than 2 of these factors had a median OS of 11.50 months. For elderly lung cancer patients without EGFR mutations, clinical parameters were the most important factors affecting survival, rather than the types of drugs.

\section{Introduction}

Lung cancer is the most common cause of cancer-associated mortality, and according to the data from western countries, $>50 \%$ of newly diagnosed patients with lung cancer are $>70$ years of age (1). With the advances in diagnostics and cancer treatment, an increasing number of elderly patients are being diagnosed with lung cancer and actively receiving treatment.

Second-line treatments are comparably efficacious in elderly as well as younger patients. For patients not harboring an epidermal growth factor receptor (EGFR) mutation, tyrosine kinase inhibitor (TKI) and cytotoxic chemotherapeutic agents are currently second-line treatment options. In a retrospective analysis performed on elderly patients ( $\geq 70$ years old) enrolled in the BR.21 trials, elderly patients revealed a benefit from second-line erlotinib treatment compared with the best supportive care with similar overall survival (OS) and response rates to those of younger patients (2). Regarding cytotoxic chemotherapy, pemetrexed or docetaxel as second-line treatment demonstrated comparable efficacy and toxicity in elderly, and younger patients in a retrospective analysis of large randomized clinical trials (3).

In addition, predicting which elderly patients may or may not benefit from chemotherapy and investigating suitable chemotherapeutics is important. Treatment without these considerations cause unnecessary treatment-associated 
toxicities, longer hospital stays, poor quality of life, economic burden and ultimately shorter survival times. As the majority of clinical trials have been performed in young and otherwise healthy people, there is insufficient knowledge regarding the efficacy and safety of drugs in elderly patients who are particularly prone to exclusion from clinical trials owing to comorbidities, coexisting multi-pharmacies or poor performance status (PS).

Comorbidities are assessed using the Charlson comorbidity index (CCI) and the simplified comorbidity score (SCS) (4-7). The CCI is one of the most widely used scoring systems and has been validated in a number of diseases and the SCS has been designed for lung cancer $(4,7)$. In addition, there are no data comparing the efficacy of TKI and chemotherapeutic drugs in elderly patients without an EGFR mutation. Therefore, the present study compared the efficacy and toxicity of these two types of drugs in elderly patients.

\section{Materials and methods}

Patients. Elderly patients ( $\geq 70$ years of age) with wild-type EGFR NSCLC who previously experienced chemotherapy failure and received second-line chemotherapy at the Korea University Guro Hospital (Seoul, Korea) between January 2005 and December 2013 were included in this retrospective study. For squamous cell carcinoma, EGFR mutation testing was not a routine practice due to a lack of possibility for $E G F R$ mutation positivity (8). Therefore, the present study included patients with squamous cell carcinoma with unknown $E G F R$ mutation status and confirmed wild-type EGFR. For other types of histology, only patients confirmed to have wild-type $E G F R$ were included. EGFR mutation status was confirmed using direct sequencing or a peptide nucleic acid (PNA) clamping method using the PNA Clamp ${ }^{\text {TM }}$ EGFR Mutation Detection kit (Panagene, Inc., Daejeon, Korea). Data were collected from electronic medical records. The present study was approved by the Institutional Review Board of the Korea University Guro Hospital (KUGH15250-001).

Between January 2005 and December 2013, 943 patients with lung cancer received palliative chemotherapy at Korea University Guro Hospital. Among them, 365 patients were $>70$ years of age and 203 of these patients received second-line chemotherapy. Following exclusion of patients harboring an EGFR mutation, 126 patients were included in the analysis. The patient characteristics are presented in Table I. The median age was 75 (range, 70-85) years. There were 101 males $(80.2 \%)$ and 25 females (19.8\%). Of the 126 patients, $58(46.0 \%)$ were diagnosed with adenocarcinoma, $63(50.0 \%)$ were diagnosed with squamous cell carcinoma and $5(4.0 \%)$ were diagnosed with another histology (3 with undifferentiated carcinoma, 1 with sarcomatoid carcinoma and 1 with large-cell carcinoma).

Data collection. The following data were collected and analyzed for each patient: Age, sex, tumor histology, stage at diagnosis, site(s) of metastasis, comorbidities, previous chemotherapy prior to second-line treatment, Eastern Cooperative Oncology Group (ECOG) PS, plasma hemoglobin levels, serum sodium and albumin levels at the beginning of the second-line chemotherapy, difference in body surface area difference (BSA) between day 1 of first-line chemotherapy and day 1 of second-line chemotherapy and the subsequent treatment following failure of second-line chemotherapy.

In addition, the present study analyzed the following data: Type of chemotherapy regimen, start and end dates of chemotherapy and response and best response to the second-line chemotherapy according to the Response Evaluation Criteria in Solid Tumors version 1.1 guidelines (9). The responses of all patients were assessed at 2- to 3-month intervals by enhanced computed tomography. Magnetic resonance imaging, fluorodeoxyglucose positron emission tomography and bone scanning were performed at the physician's discretion. Treatment-related toxicity data were collected via medical records based on the National Cancer Institute Common Terminology Criteria for Adverse Events version 3 (10).

Comorbidity assessment. For assessment of comorbidities, the present study investigated the CCI and SCS at the day of second-line chemotherapy initiation. The CCI has 19 and the SCS has 7 different descriptors, with a maximum score of 35 for the CCI, and 20 for the SCS (Tables II and III) $(4,7)$. The presence of comorbidities was assessed by reviewing electronic medical charts for individual descriptors of both comorbidity-scoring systems.

Chemotherapy regimens. Chemotherapy regimens were classified as TKI or chemotherapy, with the TKI group including patients who received an EGFR TKI, and the chemotherapy group including patients who received any type of cytotoxic chemotherapy regimen. EGFR TKIs included erlotinib and gefitinib and the cytotoxic chemotherapy regimen included docetaxel, pemetrexed, a combination of gemcitabine and vinorelbine, and platinum doublets.

Statistical analyses. All patients who received at least 1 cycle of second-line chemotherapy were included in the efficacy analysis. Rates were compared using the $\chi^{2}$ test. The Kaplan-Meier method was used to estimate the OS and progression-free survival (PFS). PFS was evaluated from the initiation of the second-line chemotherapy until the first occurrence of progression, mortality from any cause or the final follow-up visit if none of the preceding events had occurred. OS was determined as the interval between the first day of first-line treatment and mortality or the final follow-up visit. Differences between the curves were analyzed using the log-rank test. Following univariate analyses using the Kaplan-Meier method, variables significantly associated with poor survival time $(\mathrm{P}<0.05)$ were selected, and a Cox proportional hazards regression was performed for multivariate analyses using the 'ENTER' method in SPSS. SPSS for Windows version 20.0 (IBM Corp., Armonk, NY, USA) was used for all statistical analyses. $\mathrm{P}<0.05$ was considered to indicate a statistically significant difference.

\section{Results}

Patient characteristics. Among the 126 patients included in the present study, $52(41.3 \%)$ were treated with TKIs and $74(58.7 \%)$ were treated with cytotoxic chemotherapy. In the TKI group, 21 patients $(40.4 \%)$ were treated with 
Table I. Patient characteristics.

\begin{tabular}{|c|c|c|c|c|}
\hline Characteristics & All patients & TKI & Chemotherapy & P-value ${ }^{a}$ \\
\hline No. patients $(\%)$ & $126(100.0)$ & $52(41.3)$ & $74(58.7)$ & \\
\hline Age, years (range) & $75(70-85)$ & $73(70-85)$ & $75(70-81)$ & \\
\hline Age, years $(\%)$ & & & & 0.456 \\
\hline$<75$ & $80(63.5)$ & $35(67.3)$ & $45(60.8)$ & \\
\hline$\geq 75$ & $46(36.5)$ & $17(32.7)$ & $29(39.2)$ & \\
\hline $\operatorname{Sex}(\%)$ & & & & 0.845 \\
\hline Male & $101(80.2)$ & $42(80.8)$ & $59(79.7)$ & \\
\hline Female & $25(19.8)$ & $10(19.2)$ & $15(20.3)$ & \\
\hline Histology (\%) & & & & $<0.001$ \\
\hline Non-squamous & $63(50.0)$ & $38(73.1)$ & $25(33.8)$ & \\
\hline Squamous & $63(50.0)$ & $14(26.9)$ & $49(66.2)$ & \\
\hline Smoking history $(\%)$ & & & & 0.413 \\
\hline Never smoker & $27(21.4)$ & $13(25.0)$ & $14(18.9)$ & \\
\hline Ever smoker & $99(78.6)$ & $39(75.0)$ & $60(81.1)$ & \\
\hline Stage at diagnosis $(\%)$ & & & & 0.360 \\
\hline I-IIIA & $22(17.5)$ & $11(21.2)$ & $11(14.9)$ & \\
\hline IIIB-IV & $104(82.5)$ & $41(78.8)$ & $63(85.1)$ & \\
\hline Stage at second-line chemotherapy $(\%)$ & & & & 0.549 \\
\hline M0/M1a & $79(62.7)$ & $31(59.6)$ & $48(64.9)$ & \\
\hline M1b & $47(37.3)$ & $21(40.4)$ & $26(35.1)$ & \\
\hline PFS of first-line line chemotherapy (\%) & & & & 0.383 \\
\hline$<4$ months & $64(50.8)$ & $24(46.2)$ & $40(54.1)$ & \\
\hline$\geq 4$ months & $62(49.2)$ & $28(53.8)$ & $34(45.9)$ & \\
\hline ECOG PS at second-line chemotherapy (\%) & & & & 0.412 \\
\hline $0-1$ & $87(69.0)$ & $38(73.1)$ & $49(66.2)$ & \\
\hline $2-3$ & $39(31.0)$ & $14(26.9)$ & $25(33.8)$ & \\
\hline Subsequent treatment following failure of second-line chemotherapy (\%) & & & & 0.036 \\
\hline Second-line & $51(40.5)$ & $15(28.8)$ & $36(48.6)$ & \\
\hline Third-line & $39(31.0)$ & $22(42.3)$ & $17(23.0)$ & \\
\hline Fourth-line or more & $36(28.6)$ & $15(28.8)$ & $21(28.4)$ & \\
\hline BSA change between first and second-line treatment $(\%)$ & & & & 0.963 \\
\hline Not decreased & $73(57.9)$ & $30(57.7)$ & $43(58.1)$ & \\
\hline Decreased & $53(42.1)$ & $22(42.3)$ & $31(41.9)$ & \\
\hline Plasma $\mathrm{Hb}$ at the second-line chemotherapy, g/dl (\%) & & & & 0.153 \\
\hline$<10$ & $43(34.1)$ & $14(26.9)$ & $29(39.2)$ & \\
\hline$\geq 10$ & $83(65.9)$ & $38(45.8)$ & $45(54.2)$ & \\
\hline Serum sodium at the second-line chemotherapy, $\mathrm{mmol} / \mathrm{l}(\%)$ & & & & 0.482 \\
\hline$<135$ & $68(54.0)$ & $30(57.7)$ & $38(51.4)$ & \\
\hline$\geq 135$ & $58(46.0)$ & $22(42.3)$ & $36(48.6)$ & \\
\hline Serum albumin at the second-line chemotherapy, g/dl (\%) & & & & 0.224 \\
\hline$<3.5$ & $25(19.8)$ & $13(25.0)$ & $23(16.2)$ & \\
\hline$\geq 3.5$ & $101(80.2)$ & $39(75.0)$ & $62(83.8)$ & \\
\hline
\end{tabular}

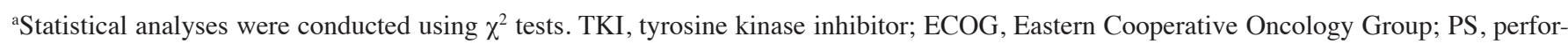
mance status; PFS; progression-free survival; SCS, simplified comorbidity score; BSA, body surface area; Hb, hemoglobin; HR, hazard ratio; CI, confidence interval.

gefitinib and $31(59.6 \%)$ were treated with erlotinib. In the chemotherapy group, docetaxel was the most common agent, which was administered to 34 patients (45.9\%). Pemetrexed was administered to 20 patients $(27.0 \%)$. A total of 9 patients 
$(12.2 \%)$ were treated with a combination of gemcitabine and vinorelbine, and 7 patients $(9.4 \%)$ were treated with a platinum doublet combination. A total of 4 patients $(5.4 \%)$ were treated with a singlet agent other than docetaxel or pemetrexed ( 3 with gemcitabine and 1 with vinorelbine). A greater percentage of patients had non-squamous cell carcinoma in the TKI group, and more patients had squamous cell carcinoma in the chemotherapy group $(\mathrm{P}<0.001)$. The number of patients who received further treatment was not balanced between the 2 treatment groups. In the TKI group, $71.1 \%$ of patients received subsequent chemotherapy following failure of second-line treatment. Conversely, $51.4 \%$ of patients in the chemotherapy group received further treatment, including patients who received more than third-line chemotherapy $(\mathrm{P}=0.036)$. Except for histology and subsequent chemotherapy, there were no significant differences in the clinical characteristics of the patients between the two treatment groups (Table I).

Comorbidities of patients. The distribution of comorbidities in the included patients is presented in Table IV. A total of 69 patients $(54.8 \%)$ had hypertension, $33(26.2 \%)$ had diabetes and $46(36.5 \%)$ had chronic pulmonary disease, including chronic obstructive pulmonary disease and interstitial lung disease. A history of solid tumor prior to second-line chemotherapy for lung cancer was observed in 15 patients; all cases were localized tumors. There were no patients with hemiplegia, dementia or acquired immune deficiency syndrome. A total of 20 patients $(15.9 \%)$ had no underlying disease except metastatic lung cancer. The CCI scores of all patients ranged between 9 and 12 (median, 10) points. The SCS of all patients ranged between 1 and 15 (median, 8) points. There were no significant differences in the distribution of CCI scores and SCSs between the TKI, and chemotherapy groups.

Clinical parameters associated with treatment outcomes. The median PFS and OS for all patients who received second-line treatment was 2.47 months [95\% confidence interval (CI), 2.08-2.86] and 8.63 months (95\% CI, 5.99-11.28), respectively (Fig. 1A). Univariate analysis demonstrated that the OS for all patients was significantly associated with the following factors: Histology (squamous vs. non-squamous), stage at diagnosis (stage I-IIIA vs. stage IIIB-IV), PS (0-1 vs. 2-3), SCS ( $<13$ vs. $\geq 13$ ), PFS of first-line treatment ( $<4$ months vs. $\geq 4$ months), change of BSA between first-line and second-line treatment (not decreased vs. decreased), subsequent treatment after failure of second-line chemotherapy (at least third-line chemotherapy vs. no chemotherapy), brain metastasis (no vs. yes), distant lymph node metastasis (no vs. yes), number of metastatic organs ( $<3 \mathrm{vs} . \geq 3$ ), plasma hemoglobin at the start of second-line chemotherapy ( $<10 \mathrm{vs} . \geq 10 \mathrm{~g} / \mathrm{dl})$, serum albumin at the start of second-line chemotherapy $(<3.5 \mathrm{vs}$. $\geq 3.5 \mathrm{~g} / \mathrm{dl})$ and serum sodium at the start of second-line chemotherapy $(<135$ vs. $\geq 135 \mathrm{mmol} / \mathrm{l})$. CCI, response to second-line treatment or adrenal gland, liver or bone metastasis were not associated with OS. The present study then performed multivariate analysis using a Cox proportional hazards regression model. Histology (squamous vs. non-squamous), good PS (ECOG 0-1), lower SCS $(<13)$, no brain metastasis, longer PFS of previous chemotherapy ( $\geq 4$ months), higher serum sodium level ( $>135 \mathrm{mmol} / \mathrm{l})$
Table II. Charlson comorbidity index and weighting of comorbidities.

\begin{tabular}{ll}
\hline Score & \multicolumn{1}{c}{ Comorbid condition } \\
\hline 1 & Myocardial infarction \\
& Congestive heart failure \\
& Cerebral vascular disease \\
& Peripheral vascular disease \\
& Dementia \\
& Chronic pulmonary disease \\
& Connective tissue disease \\
& Peptic ulcer disease \\
& Mild liver disease \\
& Age \\
& Diabetes \\
& Hemiplegia \\
& Moderate/severe renal disease \\
& Diabetes with end-organ damage \\
& Any solid tumor \\
& Leukemia \\
& Lymphoma \\
& Moderate/severe liver disease \\
& Metastatic solid tumor \\
& Acquired immunodeficiency syndrome \\
&
\end{tabular}

Scores were obtained using the Charlson comorbidity index (4). ${ }^{\mathrm{a}} \mathrm{For}$ each decade after 40 years, a point is added (1 point for age group 41-50, 2 points for age group 51-60, 3 points for $61-70$ and 6 points for 71 or older).

Table III. Simplified comorbidity score and weighting of comorbidities

\begin{tabular}{ll}
\hline Score & \multicolumn{1}{c}{ Comorbidity } \\
\hline 7 & Tobacco consumption \\
5 & Diabetes mellitus \\
4 & Renal insufficiency \\
1 & Respiratory comorbidity \\
1 & Neoplastic comorbidity \\
1 & Cardiovascular comorbidity \\
1 & Alcoholism \\
\hline
\end{tabular}

Comorbidities were assessed according to the simplified comorbidity score (7).

and higher serum albumin level (>3.5 g/dl) were associated with prolonged OS (Table V).

The present study subsequently performed survival analysis of patients who exhibited poor prognostic factors to identify patients who did not benefit from second-line chemotherapy. As the present study surmised that histology was not yet validated as prognostic markers to decide second-line treatment, a subgroup was constructed that did not include histology, but 
Table IV. Comorbidities and comorbidity scores of the patients included in the present study.

\begin{tabular}{|c|c|c|}
\hline Cormorbity & Number & $\%$ \\
\hline \multicolumn{3}{|l|}{ Comorbidities } \\
\hline Diabetes mellitus & 33 & 26.2 \\
\hline Chronic pulmonary disease & 46 & 36.5 \\
\hline Congestive heart failure & 5 & 4.0 \\
\hline Cerebrovascular disease & 9 & 7.1 \\
\hline Peripheral vascular disease & 10 & 7.9 \\
\hline Ulcer disease & 6 & 4.8 \\
\hline Myocardial infarction & 5 & 4.0 \\
\hline Liver disease & 3 & 2.4 \\
\hline Chronic kidney disease & 2 & 1.6 \\
\hline None except lung cancer & 20 & 15.9 \\
\hline \multicolumn{3}{|l|}{ Charlson comorbidity index } \\
\hline 9 & 38 & 30.2 \\
\hline 10 & 56 & 44.4 \\
\hline 11 & 21 & 16.7 \\
\hline 12 & 11 & 8.7 \\
\hline Total & 126 & 100 \\
\hline \multicolumn{3}{|l|}{ Simplified comorbidity score } \\
\hline 1 & 12 & 9.5 \\
\hline 2 & 3 & 2.4 \\
\hline 3 & 6 & 4.8 \\
\hline 6 & 5 & 4.0 \\
\hline 7 & 1 & 0.8 \\
\hline 8 & 53 & 42.1 \\
\hline 9 & 18 & 14.3 \\
\hline 13 & 13 & 10.3 \\
\hline 14 & 10 & 7.9 \\
\hline 15 & 5 & 4.0 \\
\hline Total & 126 & 100 \\
\hline
\end{tabular}

Comorbidities were assessed according to Charlson's comorbidity index and the simplified comorbidity score $(4,7)$.

prognostic factors showing statistical significance in the Cox proportional hazards regression model were weighted 1 score for each factor. The present study then divided the patients into 2 groups: Low-risk and high-risk. The low-risk group included patients who had $\leq 2$ factors and the high-risk group included those who had $\geq 3$ factors. There were 26 patients (20.6\%) in the high-risk group. The median OS of patients in the low-risk group who received second-line treatment was 11.50 months (95\% CI, 7.80-15.20), whereas the median OS of the high-risk group was 1.73 months (95\% CI, 0.78-2.69). The difference in survival was statistically significant $(\mathrm{P}<0.001$; Fig. 1B).

Efficacy of TKI and chemotherapy. The results of efficacy analysis are presented in Table VI. Of the 126 patients, there was no case of complete remission. The best responses were partial remission (PR) in 11 patients $(15.5 \%)$ and stable disease (SD) in 51 patients (40.5\%). In the TKI group, no
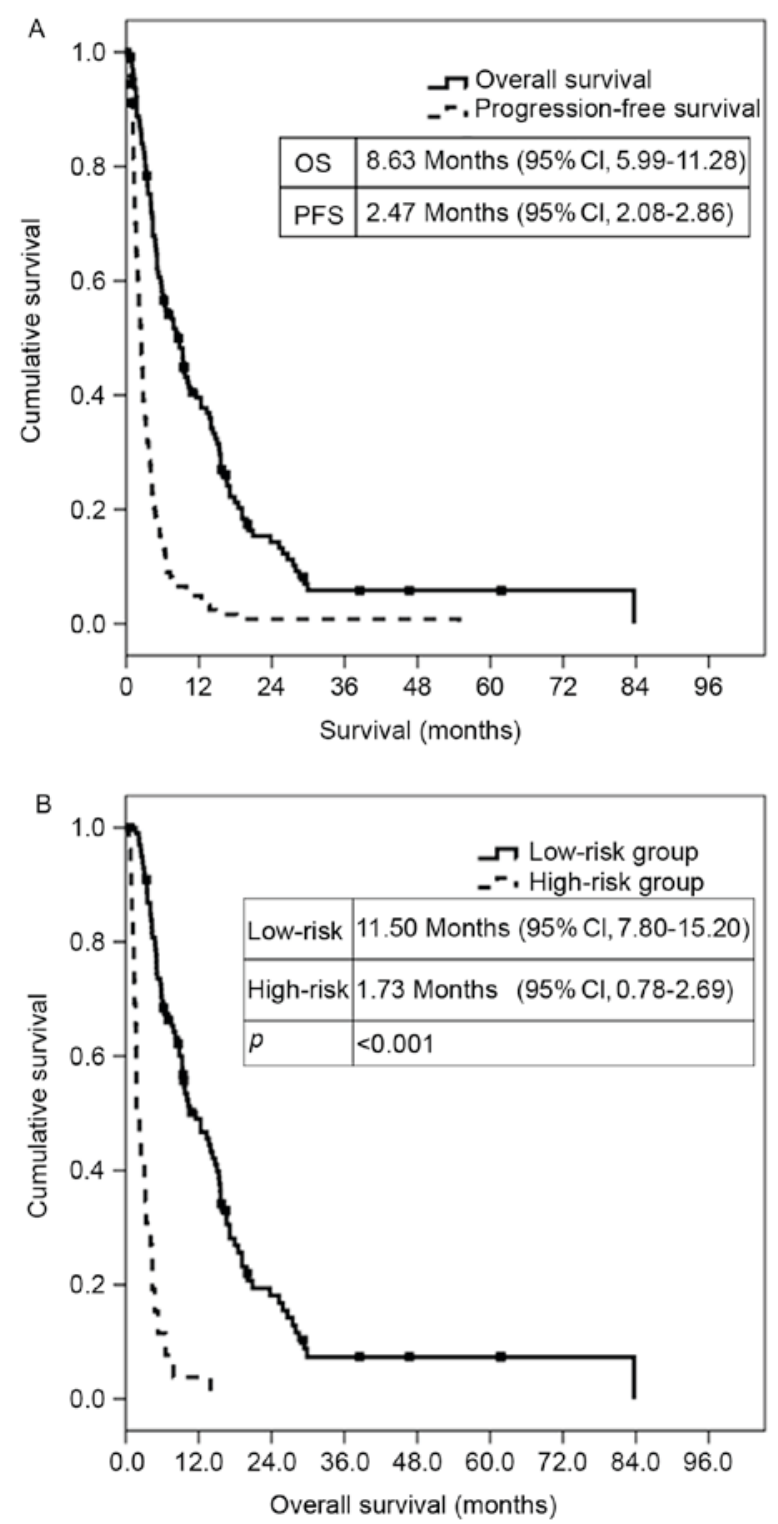

Figure 1. Survival of patients. (A) Overall survival and progression-free survival of second-line treatment in all patients. (B) Overall survival of second-line treatment according to the risk group. CI, confidence interval; OS, overall survival; PFS, progression-free survival.

patient exhibited PR. A total of 25 patients $(50.0 \%)$ revealed $\mathrm{SD}$ as the best response. However, 11 patients (15.5\%) demonstrated PR and 26 patients (36.6\%) demonstrated SD in the chemotherapy group. Therefore, the overall response rate, which is defined as the proportion of $\mathrm{PR}$ and $\mathrm{CR}$, was statistically different between the two groups $(\mathrm{P}=0.029)$.

No difference was observed in the median PFS and OS of second-line chemotherapy between the TKI, and chemotherapy groups ( $\mathrm{P}=0.287$ for $\mathrm{PFS}$ and $\mathrm{P}=0.374$ for $\mathrm{OS})$. The survival curves for PFS and OS are presented in Fig. 2.

Drug delivery and toxicities. The median treatment duration was 3.8 months. Of the 126 patients, 32 patients (25.4\%) underwent dose reduction during second-line chemotherapy, 6 in the TKI group and 26 in the chemotherapy group. A total of 13 patients $(10.3 \%)$ underwent initial dose reduction. The causes of dose reduction were chemotherapy-associated toxicity $(n=18)$ 
Table V. Multivariate analysis of prognostic factors.

All patients

Factor

\begin{tabular}{ccr}
\hline HR & $95 \%$ CI & P-value $^{\text {a }}$ \\
\hline 2.139 & $1.348-3.395$ & 0.001 \\
3.177 & $1.288-7.839$ & 0.012 \\
2.860 & $1.837-4.452$ & $<0.001$ \\
3.228 & $1.685-6.186$ & $<0.001$ \\
1.802 & $1.029-3.156$ & 0.039 \\
1.776 & $1.502-2.996$ & 0.031 \\
1.908 & $1.206-3.017$ & 0.006 \\
2.229 & $0.968-5.135$ & 0.060 \\
1.561 & $0.923-2.641$ & 0.097 \\
0.475 & $0.184-1.226$ & 0.124 \\
0.865 & $0.490-1.527$ & 0.617 \\
1.278 & $0.819-1.996$ & 0.280 \\
1.265 & $0.809-1.978$ & 0.303 \\
0.983 & $0.613-1.575$ & 0.942
\end{tabular}

ECOG PS (0-1 vs. 2-3)

Brain metastasis (negative vs. positive)

PFS of first-line treatment, months ( $\geq 4$ vs. $<4)$

Serum sodium, mmol/l ( $\geq 135$ vs. $<135)$

Serum albumin, g/dl ( $\geq 3.5$ vs. $<3.5)$

SCS ( $<13$ vs. $\geq 13$ )

Histology (non-squamous vs. squamous)

Distant lymph node metastasis (negative vs. positive)

Metastatic organ (M1a vs. M1b)

Number of metastasis organs ( $<3$ vs. $\geq 3$ )

Stage at diagnosis (I-IIIA vs. IIIB-IV)

Subsequent treatment (third-line or more chemotherapy vs. no chemotherapy)

BSA change between first- and second-line treatment (not decreased vs. decreased)

Plasma Hb, g/dl ( $\geq 10$ vs. $<10)$

${ }^{\mathrm{a} C o x}$ proportional hazards regression was conducted for multivariate analyses. ECOG, Eastern Cooperative Oncology Group; PS, performance status; PFS, progression-free survival; SCS, simplified comorbidity score; BSA, body surface area; Hb, hemoglobin; HR, hazard ratio; CI, confidence interval.

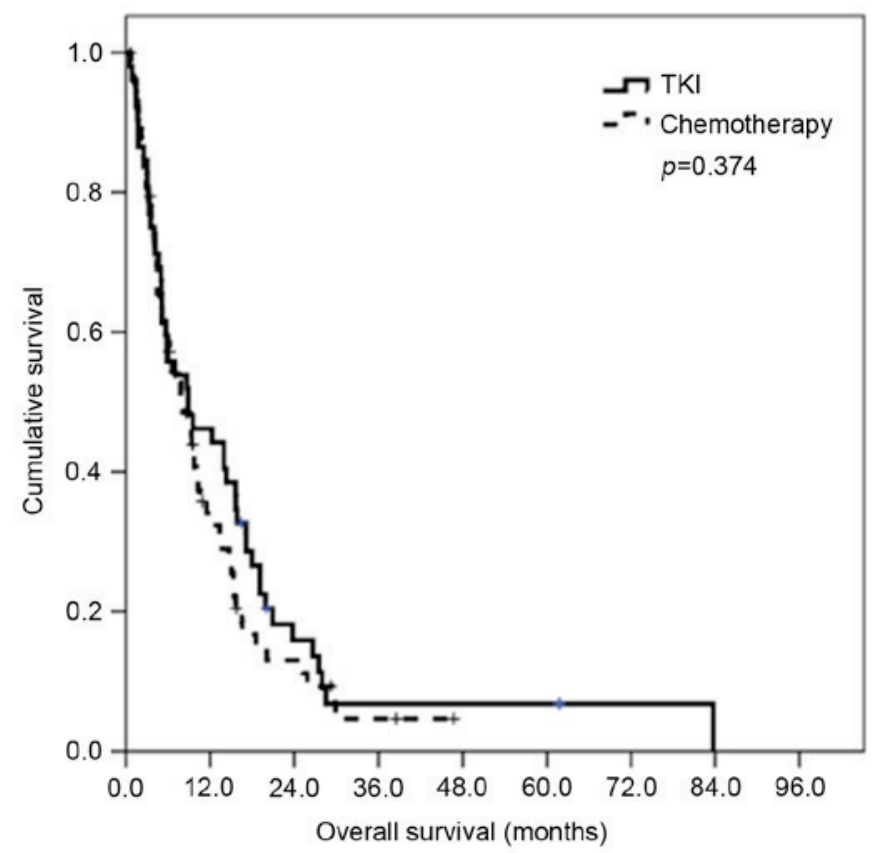

Figure 2. Overall survival of second-line treatment in TKI and chemotherapy groups. TKI, tyrosine kinase inhibitor.

and decreased PS $(n=11)$. A total of 7 patients $(5.5 \%)$ stopped chemotherapy owing to toxicity and 7 patients (5.5\%) stopped treatment due to decreased PS. A total of 3 patients $(2.4 \%)$ stopped chemotherapy because they refused further treatment. A total of 6 patients (11.5\%) in the TKI group and 7 patients $(9.4 \%)$ in the chemotherapy group stopped chemotherapy because of toxicity and poorer PS.
The frequencies of hematologic and non-hematologic adverse events are presented in Table VII. In the TKI group, non-hematological toxicities, including skin rash $(54.9 \%)$, emesis $(27.5 \%)$ and fatigue (27.5\%), were common. In terms of hematological toxicities, anemia (47.1\%) was the most common. Grade 3/4 toxicities included anemia, neutropenia, thrombocytopenia, diarrhea, emesis and fatigue, each occurring in $<3.9 \%$ of patients. In the chemotherapy group, hematological toxicities were more common. Anemia (85.1\%), neutropenia (36.5\%), thrombocytopenia (16.2\%) and febrile neutropenia (10.8\%) were observed. Regarding non-hematological toxicities, emesis and fatigue were common, occurring in 44.6 , and $41.9 \%$ of patients, respectively. Grade 3/4 toxicities included neutropenia (20.3\%), febrile neutropenia $(10.8 \%)$, anemia $(8.1 \%)$, thrombocytopenia, $(8.1 \%)$, emesis $(6.8 \%)$ and fatigue $(6.8 \%)$. There were no treatment-associated mortalities in either group.

\section{Discussion}

In the present study, clinical factors, including comorbid conditions is the most important factor affecting survival in elderly patients with lung cancer without EGFR mutations receiving second-line treatment. Additionally, clinical factors, including PS, PFS of first-line chemotherapy, presence of brain metastasis, serum albumin levels and serum sodium levels, were strong prognostic factors for elderly patients in the present study. A good PS is a well-known prognostic factor and a long PFS of previous chemotherapy reflects the less aggressive nature of the cancer $(3,11)$. Brain metastasis is also a well-known risk factor with a poor outcome. In particular, brain metastasis in elderly patients may affect tolerability to 
Table VI. Efficacy analysis of TKI and chemotherapy groups.

\begin{tabular}{lcc}
\hline Factor & TKI $(\mathrm{n}=50)$ & Chemotherapy $(\mathrm{n}=73)$ \\
\hline Response to second-line chemotherapy, $\mathrm{n}=123^{\mathrm{a}}(\%)$ & & \\
$\begin{array}{l}\text { Complete remission } \\
\text { Partial remission }\end{array}$ & $0(0.0)$ & $0(0.0)$ \\
Stable disease & $0(0.0)$ & $11(15.5)$ \\
Progressive disease & $25(50.0)$ & $26(36.6)$ \\
Response rate & $25(50.0)$ & $15.5 \%$ \\
Disease control rate & $0.0 \%$ & $52.1 \%$ \\
Survival of second-line chemotherapy & $50.0 \%$ & \\
Progression-free survival (months), median $(95 \% \mathrm{CI})$ & & $2.33(1.75-2.92)$ \\
Overall survival (months), median $(95 \% \mathrm{CI})$ & $2.57(2.18-3.00)$ & $7.83(4.80-10.87)$ \\
\hline
\end{tabular}

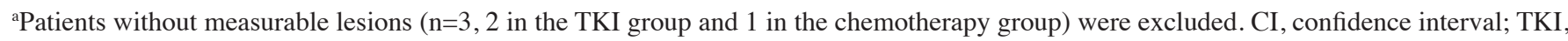
tyrosine kinase inhibitor.

Table VII. Treatment-associated adverse events of TKI and chemotherapy groups.

\begin{tabular}{|c|c|c|c|c|c|c|c|c|}
\hline \multirow[b]{3}{*}{ Adverse event } & \multicolumn{4}{|c|}{ TKI (n=52) } & \multicolumn{4}{|c|}{ Chemotherapy $(\mathrm{n}=74)$} \\
\hline & \multicolumn{2}{|c|}{ All } & \multicolumn{2}{|c|}{ Grade 3-4 } & \multicolumn{2}{|c|}{ All } & \multicolumn{2}{|c|}{ Grade 3-4 } \\
\hline & $\mathrm{n}$ & $(\%)$ & $\mathrm{n}$ & $(\%)$ & $\mathrm{n}$ & $(\%)$ & $\mathrm{n}$ & $(\%)$ \\
\hline \multicolumn{9}{|l|}{ Hematologic } \\
\hline Anemia & 24 & 47.1 & 1 & 2.0 & 63 & 85.1 & 6 & 8.1 \\
\hline Neutropenia & 1 & 2.0 & 1 & 2.0 & 27 & 36.5 & 15 & 20.3 \\
\hline Thrombocytopenia & 1 & 2.0 & 1 & 2.0 & 12 & 16.2 & 6 & 8.1 \\
\hline Febrile neutropenia & 0 & 0.0 & 0 & 0 & 8 & 10.8 & 8 & 10.8 \\
\hline \multicolumn{9}{|l|}{ Non-hematologic } \\
\hline Diarrhea & 10 & 19.6 & 1 & 2.0 & 6 & 8.1 & 0 & 0 \\
\hline Emesis & 14 & 27.5 & 1 & 2.0 & 33 & 44.6 & 5 & 6.8 \\
\hline Constipation & 1 & 2.0 & 0 & 0 & 6 & 8.1 & 0 & 0 \\
\hline Fatigue & 14 & 27.5 & 2 & 3.9 & 31 & 41.9 & 5 & 6.8 \\
\hline Neuropathy & 0 & 0 & 0 & 0 & 3 & 4.1 & 0 & 0 \\
\hline Skin rash & 28 & 54.9 & 1 & 2.0 & 1 & 1.4 & 0 & 0 \\
\hline ILD & 2 & 3.9 & 1 & 2.0 & 0 & 0 & 0 & 0 \\
\hline
\end{tabular}

TKI, tyrosine kinase inhibitor; ILD, interstitial lung disease; $n$, number.

chemotherapy, quality of life and neurotoxicities depending on the surgery or radiotherapy of the brain $(12,13)$. Furthermore, numerous studies evaluated low serum albumin and sodium levels as independent poor prognostic factors in patients with cancer (14-16). Serum albumin is a commonly used marker for assessing a patient's nutritional status, inflammation and hepatic function. Serum albumin is decreased in cases of malnutrition due to cancer cachexia, treatment-associated poor oral intake or response to acute inflammation as these situations are major causes of albumin synthesis suppression (17). Hyponatremia is caused by inappropriate antidiuretic hormone secretion syndrome, diuretic drugs and salt-wasting conditions in patients with brain metastases, meningitis, and salt-losing nephropathy following cisplatin use (16). Furthermore, poor oral intake accompanying hypovolemia may induce hyponatremia in numerous patients with cancer (16). Therefore, serum albumin and sodium are reliable factors representing nutritional status, inflammatory conditions or cancer activities. Clinicians should pay close attention to these laboratory findings when treating elderly patients with cancer.

In the present study, patients with non-squamous cell histology without an EGFR mutation demonstrated better survival, compared with the patients with squamous cell histology. The OS in lung adenocarcinoma patients has been 
reported to be higher compared with that in patients with lung squamous cell carcinoma owing to the introduction of EGFR TKI therapy (18). However, the reasons for the survival difference between patients with lung adenocarcinoma not harboring EGFR mutations and patients with squamous cell carcinoma in the present study remain unclear. Further investigations of survival according to histology in patients with lung cancer with or without target therapy options may aid in explaining this difference.

The present study divided patients into subgroups to investigate reliable clinical factors to predict which patients do not benefit from second-line chemotherapy. Therefore, the present study identified low-risk and high-risk groups according to the six factors that revealed statistical significance in the multivariate analysis. The OS of the high-risk group revealed $\geq 3$ risk factors were 1.73 months, a very short duration compared with the OS of the low-risk group patients (11.50 months). Therefore, the present study concluded that second-line treatment is not beneficial and may even be harmful to patients with numerous risk factors.

In general, comorbid conditions of patients with cancer are associated with poorer survival $(19,20)$. The present study evaluated CCI and SCS to analyze the association of comorbidities and efficacy of chemotherapy. CCI was not associated with survival, whereas SCS was associated with OS in univariate and multivariate analyses. SCS was suggested to be more sensitive to validate comorbidities. In other studies, the SCS represented an independent prognostic factor for NSCLC as well as small-cell lung cancer $(7,21)$ and was more informative compared with CCI to predict outcomes in patients with NSCLC (7), although this is still debated. Numerous studies reported that SCS did not provide prognostic information in patients with lung cancer $(6,5)$. To more precisely predict the outcome of patients with lung cancer with underlying disease, more validation is required.

For patients with lung cancer without EGFR mutations, second-line treatment includes cytotoxic agents, such as docetaxel, pemetrexed, gemcitabine and EGFR TKIs (gefitinib and erlotinib). A number of studies reported that EGFR TKI treatment in second-line treatment had similar efficacy to cytotoxic chemotherapy in patients with NSCLC (22-25). Patients with squamous cell carcinoma or NSCLC who do not harbor an EGFR mutation revealed a benefit from TKI compared with the best supportive care as second-line treatment (25-28). However, previous meta-analyses reported that cytotoxic chemotherapy is better compared with TKI for patients without EGFR mutations (29-31).

Numerous factors other than survival alone have to be considered to select therapies for elderly patients who failed previous chemotherapy as they are regarded as having a poorer physical status compared with younger patients. Physicians should take into account a number of factors in addition to age, including the patient's life expectancy, functional status and comorbidities. Therefore, TKI is an attractive choice for elderly patients as TKIs are convenient to take and require less frequent hospital visits. Furthermore, TKIs have less severe toxicities compared with cytotoxic chemotherapeutic agents. However, the treatment choice for more than second-line treatment of elderly patients with wild-type $E G F R$ remains unclear, as there have been no reports on this patient group to date.

The present study was performed with elderly patients with NSCLC not harboring EGFR mutations in order to determine a solution for the practical treatment of these patients. In the present study, TKI produced a poor response rate compared with chemotherapy. However, PFS and OS were not different between the two treatment groups. As expected, severe toxicities exceeding grade 3 were more common in the chemotherapy group and dose reduction was also more common in the chemotherapy group, compared with the TKI group. Therefore, the results of the present study suggested that TKI may be an appropriate second-line treatment option for elderly patients. Furthermore, the present study suggested that careful dose adjustment is necessary during cytotoxic chemotherapy treatment in this age group. Hematologic toxicities are generally more common with chemotherapy compared with TKI therapy, and these toxicities affect the quality of life as well as the dose intensity during chemotherapy. In addition, elderly patients frequently have numerous comorbidities and these comorbidities also affect the dose of drugs. A low dose intensity may result in shorter survival times and hematologic toxicities may sometimes cause treatment-associated mortality. Therefore, physicians should administer well-managed chemotherapy and pay careful attention to elderly patients.

As studies of the parameters associated with treatment outcomes of second-line therapy in this clinical setting are scarce, the prognostic factors identified in the present study should be useful for designing prospective, randomized clinical trials on the efficacy of second-line chemotherapy in these patients. In addition, the present study investigated the usefulness of comorbidity scores, including the CCI and SCS, as prognostic factors. Comorbidity analysis is important in studies of elderly patients, but few such studies have been reported to date $(32,33)$. Based on the results of the present study, it is suggested that clinical factors as well as comorbidity factors have a strong predictive value with respect to survival. To the best of our knowledge, this is the first study comparing second-line TKI and chemotherapy treatment in elderly patients with NSCLC and wild-type EGFR. Although the findings were limited by the small number of patients evaluated and the retrospective study design, they provide a practical guide for selecting second-line chemotherapy in elderly patients without EGFR mutations.

Previously, novel immunotherapeutics, including programmed death ligand-1 immune check point blockade revealed high efficacy in patients with lung cancer. Nivolumab, pembrolizumab and atezolizumab demonstrated benefits in terms of survival compared with docetaxel as second-line treatment. Furthermore, these agents revealed good safety profiles (34) and therefore would be more beneficial to elderly patients. Further investigation of immunotherapeutics is required.

In conclusion, significant prognostic factors affecting survival identified in the current study were squamous histology, poor PS, higher SCS, short PFS of first-line 
treatment, presence of brain metastasis, low serum albumin level and hyponatremia. Careful consideration should be given in deciding second-line treatment for patients having $\geq 3$ of these factors, except histology. TKI and cytotoxic chemotherapy as second-line treatment revealed similar survival results and different toxicity profiles. Both are good options for elderly patients with NSCLC not harboring EGFR mutations if careful management is provided. Therefore, physicians should consider clinical conditions of each patient as the most important factors affecting survival in the second-line treatment of elderly patients.

\section{References}

1. Vora N and Reckamp KL: Non-small cell lung cancer in the elderly: Defining treatment options. Semin Oncol 35: 590-596, 2008.

2. Wheatley-Price P, Ding K, Seymour L, Clark GM and Shepherd FA: Erlotinib for advanced non-small-cell lung cancer in the elderly: An analysis of the National Cancer Institute of Canada clinical trials group study BR.21. J Clin Oncol 26: 2350-2357, 2008.

3. Weiss GJ, Langer C, Rosell R, Hanna N, Shepherd F, Einhorn LH, Nguyen B, Paul S, McAndrews P, Bunn PA Jr and Kelly K: Elderly patients benefit from second-line cytotoxic chemotherapy: A subset analysis of a randomized phase III trial of pemetrexed compared with docetaxel in patients with previously treated advanced non-small-cell lung cancer. J Clin Oncol 24: 4405-4411, 2006.

4. Charlson M, Szatrowski TP, Peterson J and Gold J: Validation of a combined comorbidity index. J Clin Epidemiol 47: 1245-1251, 1994.

5. Alexander M, Evans SM, Stirling RG, Wolfe R, Officer A, MacManus M, Solomon B, Burbury K and Ball D: The influence of comorbidity and the simplified comorbidity score on overall survival in non-small cell lung cancer-a prospective cohort study. J Thorac Oncol 11: 748-757, 2016.

6. Ball D, Thursfield V, Irving L, Mitchell P, Richardson G, Torn-Broers Y, Wright G and Giles G: Evaluation of the simplified comorbidity score (Colinet) as a prognostic indicator for patients with lung cancer: A cancer registry study. Lung Cancer 82: 358-361, 2013.

7. Colinet B, Jacot W, Bertrand D, Lacombe S, Bozonnat MC, Daurès JP and Pujol JL; oncoLR health network: A new simplified comorbidity score as a prognostic factor in non-small-cell lung cancer patients: Description and comparison with the Charlson's index. Br J Cancer 93: 1098-1105, 2005.

8. Lindeman NI, Cagle PT, Beasley MB, Chitale DA, Dacic S, Giaccone G,Jenkins RB, Kwiatkowski DJ, Saldivar JS, Squire J, et al: Molecular testing guideline for selection of lung cancer patients for EGFR and ALK tyrosine kinase inhibitors: Guideline from the college of American pathologists, international association for the study of lung cancer and association for molecular pathology. J Mol Diagn 15: 415-453, 2013.

9. Eisenhauer EA, Therasse P, Bogaerts J, Schwartz LH, Sargent D, Ford R, Dancey J, Arbuck S, Gwyther S, Mooney M, et al: New response evaluation criteria in solid tumours: Revised RECIST guideline (version 1.1). Eur J Cancer 45: 228-247, 2009.

10. National Cancer Institute: Common Terminology Criteria for Adverse Events v3.0 (CTCAE), 2006. https://ctep.cancer.gov/ protocoldevelopment/electronic_applications/docs/ctcaev3.pdf. Accessed August 9, 2006.

11. Imai H, Takahashi T, Mori K, Ono A, Akamatsu H, Shukuya T, Taira T, Kenmotsu H, Naito T, Murakami H, et al: Individual-level dataon the relationships of progression-free survival, post-progression survival, and tumor response with overall survival in patients with advanced non-squamous non-small cell lung cancer. Neoplasma 61: 233-240, 2014.

12. DeAngelis LM, Delattre JY and Posner JB: Radiation-induced dementia in patients cured of brain metastases. Neurology 39: 789-796, 1989.

13. Aoyama H, Tago M, Kato N, Toyoda T, Kenjyo M, Hirota S, Shioura H, Inomata $\mathrm{T}$, Kunieda $\mathrm{E}$, Hayakawa $\mathrm{K}$, et al: Neurocognitive function of patients with brain metastasis who received either whole brain radiotherapy plus stereotactic radiosurgery or radiosurgery alone. Int J Radiat Oncol Biol Phys 68: 1388-1395, 2007.
14. Tanriverdi O, Avci N, Oktay E, Kalemci S, Pilanci KN, Cokmert S, Menekse S, Kocar M, Sen CA, Akman T, et al: Pretreatment serum albumin level is an independent prognostic factor in patients with stage IIIB non-small cell lung cancer: A study of the turkish descriptive oncological researches group. Asian Pac J Cancer Prev 16: 5971-5976, 2015.

15. Kobayashi N, Usui S, Yamaoka M, Suzuki H, Kikuchi S, Goto Y, Sakai M and Sato Y: The influence of serum sodium concentration on prognosis in resected non-small cell lung cancer. Thorac Cardiovasc Surg 62: 338-343, 2014.

16. Castillo JJ, Vincent M and Justice E: Diagnosis and management of hyponatremia in cancer patients. Oncologist 17: 756-765, 2012.

17. Simons JP, Schols AM, Buurman WA and Wouters EF: Weight loss and low body cell mass in males with lung cancer: Relationship with systemic inflammation, acute-phase response, resting energy expenditure, and catabolic and anabolic hormones. Clin Sci (Lond) 97: 215-223, 1999.

18. Chang JS, Chen LT, Shan YS, Lin SF, Hsiao SY, Tsai CR, $\mathrm{Yu}$ SJ and Tsai HJ: Comprehensive analysis of the incidence and survival patterns of lung cancer by histologies, including rare subtypes, in the era of molecular medicine and targeted therapy: A nation-wide cancer registry-based study from Taiwan. Medicine (Baltimore) 94: e969, 2015.

19. Islam KM, Jiang X, Anggondowati T, Lin G and Ganti AK: Comorbidity and survival in lung cancer patients. Cancer Epidemiol Biomarkers Prev 24: 1079-1085, 2015.

20. Jacot W, Colinet B, Bertrand D, Lacombe S, Bozonnat MC, Daurès JP and Pujol JL; OncoLR health network: Quality of life and comorbidity score as prognostic determinants in non-small-cell lung cancer patients. Ann Oncol 19: 1458-1464, 2008.

21. Kuo YW, Jerng JS, Shih JY, Chen KY, Yu CJ and Yang PC: The prognostic value of the simplified comorbidity score in the treatment of small cell lung carcinoma. J Thorac Oncol 6: 378-383, 2011.

22. Kim ES, Hirsh V, Mok T, Socinski MA, Gervais R, Wu YL, Li LY, Watkins CL, Sellers MV, Lowe ES, et al: Gefitinib versus docetaxel in previously treated non-small-cell lung cancer (INTEREST): A randomised phase III trial. Lancet 372: 1809-1818, 2008.

23. Sun JM, Lee KH, Kim SW, Lee DH, Min YJ, Yun HJ, Kim HK, Song HS, Kim YH, Kim BS, et al: Gefitinib versus pemetrexed as second-line treatment in patients with nonsmall cell lung cancer previously treated with platinum-based chemotherapy (KCSG-LU08-01): An open-label, phase 3 trial. Cancer 118: 6234-6242, 2012

24. Garassino MC, Martelli O, Broggini M, Farina G, Veronese S, Rulli E, Bianchi F, Bettini A, Longo F, Moscetti L, et al: Erlotinib versus docetaxel as second-line treatment of patients with advanced non-small-cell lung cancer and wild-type EGFR tumours (TAILOR): A randomised controlled trial. Lancet Oncol 14: 981-988, 2013.

25. Kawaguchi T, Ando M, Asami K, Okano Y, Fukuda M, Nakagawa H, Ibata H, Kozuki T, Endo T, Tamura A, et al: Randomized phase III trial of erlotinib versus docetaxel as second- or third-line therapy in patients with advanced non-small-cell lung cancer: Docetaxel and Erlotinib Lung Cancer Trial (DELTA). J Clin Oncol 32: 1902-1908, 2014.

26. Zhao HY, Zhang Y, Huang H, Chen LK, Xu GC and Zhang L: Efficacy of erlotinib on advanced non-small cell lung cancer. Ai Zheng 27: 393-399, 2008 (In Chinese).

27. Li N, Ou W, Yang H, Liu QW, Zhang SL, Wang BX and Wang SY: A randomized phase 2 trial of erlotinib versus pemetrexed as second-line therapy in the treatment of patients with advanced EGFR wild-type and EGFR FISH-positive lung adenocarcinoma. Cancer 120: 1379-1386, 2014.

28. Ma K, Cohen V, Kasymjanova G, Small D, Novac K, Peterson J, Levit A and Agulnik J: An exploratory comparative analysis of tyrosine kinase inhibitors or docetaxel in second-line treatment of EGFR wild-type non-small-cell lung cancer: A retrospective real-world practice review at a single tertiary care centre. Curr Oncol 22: e157-e163, 2015.

29. Lee JK, Hahn S, Kim DW, Suh KJ, Keam B, Kim TM, Lee SH and Heo DS: Epidermal growth factor receptor tyrosine kinase inhibitors vs conventional chemotherapy in non-small cell lung cancer harboring wild-type epidermal growth factor receptor: A meta-analysis. JAMA 311: 1430-1437, 2014. 
30. Zhao N, Zhang XC, Yan HH, Yang JJ and Wu YL: Efficacy of epidermal growth factor receptor inhibitors versus chemotherapy as second-line treatment in advanced non-small-cell lung cancer with wild-type EGFR: A meta-analysis of randomized controlled clinical trials. Lung Cancer 85: 66-73, 2014.

31. Vale CL, Burdett S, Fisher DJ, Navani N, Parmar MK, Copas AJ and Tierney JF: Should tyrosine kinase inhibitors be considered for advanced non-small-cell lung cancer patients with wild type EGFR? Two systematic reviews and meta-analyses of randomized trials. Clin Lung Cancer 16: 173-182.e4, 2015.

32. Gironés R, Torregrosa D, Gómez-Codina J, Maestu I, Tenias JM and Rosell R: Prognostic impact of comorbidity in elderly lung cancer patients: Use and comparison of two scores. Lung Cancer 72: 108-113, 2011.
33. Haruki T, Yurugi Y, Wakahara M, Matsuoka Y, Miwa K, Araki K, Taniguchi Y and Nakamura H: Simplified comorbidity score for elderly patients undergoing thoracoscopic surgery for lung cancer. Surg Today 47: 718-725, 2017.

34. Meng X, Liu Y, Zhang J, Teng F, Xing L and Yu J: PD-1/PD-L1 checkpoint blockades in non-small cell lung cancer: New development and challenges. Cancer Lett 405: 29-37, 2017. 